\title{
Death, discharge and arrhythmias among patients with COVID-19 and cardiac injury
}

\author{
Daoyuan Si MD PhD, Beibei Du MD PhD, Lujia Ni MD, Bo Yang MD, Huan Sun MD PhD, Nan Jiang MD, \\ Guohui Liu MD, Stéphane Massé MASc, Lina Jin PhD, Jared Nanthakumar, Abhishek Bhaskaran MD PhD, \\ Ping Yang MD PhD, Kumaraswamy Nanthakumar MD
}

Cite as: CMAJ 2020 July 13;192:E791-8. doi: 10.1503/cmaj.200879; early-released June 25, 2020

See related article at www.cmaj.ca/lookup/doi/10.1503/cmaj.201230

\begin{abstract}
BACKGROUND: Cardiac injury is common in severe coronavirus disease 2019 (COVID19) and is associated with poor outcomes. We aimed to study predictors of in-hospital death, characteristics of arrhythmias and the effects of QT-prolonging therapy in patients with cardiac injury.
\end{abstract}

METHODS: We conducted a retrospective cohort study involving patients with severe COVID-19 who were admitted to Tongji Hospital in Wuhan, China, between Jan. 29 and Mar. 8, 2020. Among patients who had cardiac injury, which we defined as an elevated level of cardiac troponin I (cTnl), we identified demographic and clinical characteristics associated with mortality and need for invasive ventilation.
RESULTS: Among 1284 patients with severe COVID-19, 1159 had a cTnl level measured on admission to hospital, of whom 170 (14.7\%) had results that showed cardiac injury. We found that mortality was markedly higher in patients with cardiac injury $(71.2 \%$ v. $6.6 \%, p<0.001)$. We determined that initial cTnI (per 10 -fold increase, hazard ratio [HR] 1.32, 95\% confidence interval $[\mathrm{Cl}] 1.06-1.66$ ) and peak $\mathrm{cTn}$ l level during illness (per 10-fold increase, HR $1.70,95 \% \mathrm{Cl} 1.38-$ 2.10) were associated with poor survival. Peak cTnl was also associated with the need for invasive ventilation (odds ratio 3.02, 95\% Cl 1.92-4.98). We found arrhythmias in 44 of the 170 patients with cardiac injury (25.9\%), including 6 patients with ventricular tachycardia or fibrillation, all of whom died. We determined that patients who received QT-prolonging drugs had longer QTc intervals than those who did not receive them (difference in medians, $45 \mathrm{~ms}, p=0.01$ ), but such treatment was not independently associated with mortality (HR 1.04, 95\% Cl 0.69-1.57).

INTERPRETATION: We found that in patients with COVID-19 and cardiac injury, initial and peak cTnl levels were associated with poor survival, and peak CTnl was a predictor of need for invasive ventilation. Patients with COVID-19 warrant assessment for cardiac injury and monitoring, especially if therapy that can prolong repolarization is started. Trial registration: Chinese Clinical Trial Registry, No. ChiCTR2000031301.

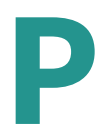

oor outcomes have been reported recently in patients with pneumonia associated with coronavirus disease 2019 (COVID-19) and cardiac injury. ${ }^{1-3}$ These reports did not characterize patients as dead or discharged from hospital because the COVID-19 pandemic had not completed its course at the time of reporting. ${ }^{1-3}$ The initial findings suggested that patients admitted to the intensive care unit (ICU) had an arrhythmia burden of $44.4 \% ;{ }^{4}$ however, the exact nature of these arrhythmias was not characterized. Knowing now that cardiac injury is an important predictor of death, characterizing arrhythmias and determining independent predictors of outcome may allow health care providers to implement aggressive therapy and assign accurate probabilities for the outcome, which can be used to identify high-risk groups. In addition, such data would assist in decisions on discharge from the emergency department, therapy with QT-prolonging drugs, rhythm monitoring and triage of ventilators and ICU beds. ${ }^{5}$
In Wuhan, China, the initial outbreak of COVID-19 has run its full course, which provides an opportunity to characterize outcomes and inform strategy for Europe and North America. As such, we evaluated 170 patients from Wuhan who had cardiac injury that was diagnosed early during their admission for pneumonia associated with COVID19 for the outcomes of death, discharge and arrhythmias. We also characterized the effect of QT-prolonging drugs in these patients. We determined independent predictors of death and mechanical ventilation in this population with cardiac injury and severe COVID-19.

\section{Methods}

\section{Study participants, setting and design}

Infection caused by severe acute respiratory syndrome coronavirus 2 (SARS-CoV-2) was diagnosed using contact history, clinical symptoms and a positive result for SARS-CoV-2 using nucleotide polymerase 
chain reaction (PCR) testing, based on the interim guidelines from the World Health Organization. ${ }^{6}$ Tongji Hospital in Wuhan, China, is one of the government-assigned hospitals that was delegated to provide regional care of patients with COVID-19. Patients transferred to this hospital had pneumonia associated with COVID-19 that was confirmed by computed tomography (CT). We reviewed information for 1284 patients who were transferred to Tongji Hospital (ZhongfaXincheng campus) from Jan. 29 to Mar. 8, 2020. The data for these patients were extracted from the hospital health records by the regional COVID-19 care coordinator (B.Y.) and a cardiologist (D.S.). These data were then reviewed by another cardiologist (B.D.) to assess their validity. The resultant records of rhythm abnormalities and electrocardiograms (ECGs) were assessed by 3 different cardiologists and consensus was reached (D.S., H.S. and B.D.).

We included 170 patients with elevated levels of serum cardiac troponin I (cTnl; levels of cTnl above the 99th percentile of the upper reference limit [ $>26.2 \mathrm{pg} / \mathrm{mL}]$ ) on hospital admission. The levels of serum cTnl were measured in each patient within 72 hours of admission. Follow-up cTnl levels were determined daily or as felt appropriate by the clinical COVID-19 team, considering the difficulties of phlebotomy in an isolated patient. The initial determination of cTnl level was separate from the peak cTnl level identified during the hospital stay. We defined the peak cTnl level as the highest cTnl concentration observed in the tests during the patient's stay in hospital. We defined onset of illness as the onset of typical symptoms related to COVID-19, such as fever, dry cough and dyspnea.

We collected data on treatment, respiratory support and arrhythmias from the patient's medical records. We identified cardiac arrhythmias based on the diagnosis made by the COVID-19 team cardiologist as documented in the patient's chart. We confirmed these findings through an independent review of the recordings by some of the investigators who are cardiac electrophysiologists (D.S., B.D., A.B. and K.N.). Telemetry monitoring was mainly used for the recordings because only a few patients had a 12-lead ECG documented in their chart owing to the need to minimize exposure of health care workers to SARS-CoV-2. We classified rhythm diagnoses as frequent premature atrial complexes (fPACs), atrial tachycardia/atrial fibrillation (AT/AF), frequent premature ventricular complexes (fPVCs) or ventricular tachycardia/ventricular fibrillation (VT/VF). We defined FPAC and fPVC as 3 or more PACs or PVCs in 10 continuous beats on the monitor or ECG. We obtained the direct causes of death for each patient from the final clinical records.

We quantified the effect of QT-prolonging antiviral and antibiotic drugs in patients with COVID-19 who had ECGs. We classified moxifloxacin, levofloxacin, azithromycin, chloroquine and lopinavir as QT-prolonging drugs, and oseltamivir, ribavirin, arbidol, ganciclovir, cefoperazone, biapenem, meropenem and imipenem as drugs that do not prolong QT.

\section{Statistical analysis}

We expressed continuous variables as medians $\left(P_{25}, P_{75}\right)$. We employed the Mann-Whitney $U$ test to compare the medians between groups. Categorical variables were expressed as numbers and percentages. We used the Fisher exact test to compare the percentages. In the timeto-event analysis, we used in-hospital death as the outcome; we excluded patients who were discharged. We calculated hazard ratios
(HRs) and 95\% confidence intervals (Cls) for peak cTnl values using the Cox regression model, where log-transformation of peak cTnl was applied, and age and sex (female) were adjusted for usage of QT prolongation medication in this multivariable analysis. We categorized peak cTnl values into tertiles and tested for trend using the Wald test of the continuous variable set to the median values of tertiles of the peak cTnl level. We used GraphPad Prism 7.00 software (San Diego, California) and R version 3.6.3 (University of Auckland, Oakland, New Zealand) for data analysis. All tests were 2-sided, and we considered a value of $p<0.05$ to be statistically significant.

\section{Ethics approval}

This study (ChiCTR2000031301) was approved by the Institutional Research Ethics Boards of the Third Hospital of Jilin University (approval no. 2020032619), Tongji Hospital of Huazhong University of Science and Technology (approval no. TJ-IRB20200345) and the University Health Network, Toronto, Canada (REB no. 20-5322).

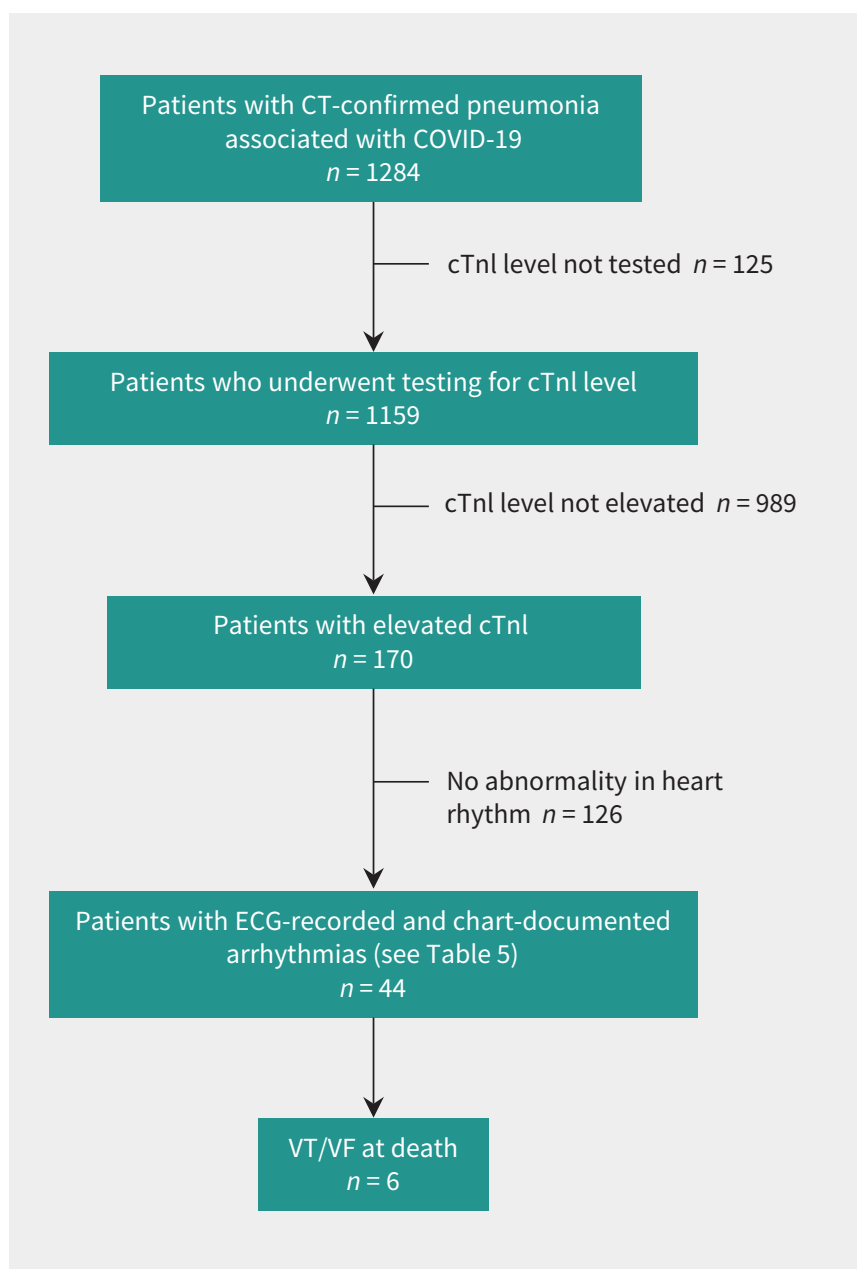

Figure 1: Flow chart of the study population. Patients $(n=1284)$ with pneumonia associated with coronavirus disease 2019 (COVID-19) that was confirmed by computed tomography (CT) were transferred to Tongji Hospital, Wuhan, China, and included in our study. From this group, 1159 patients underwent testing for cardiac troponin I (cTnl) at admission to hospital, of which 170 had a positive result (elevated level of cTnI). Of these 170 patients, 44 had abnormalities in heart rhythm diagnosed, and 6 died of ventricular tachycardia/ventricular fibrillation (VT/VF). Note: ECG = electrocardiogram. 


\section{Results}

In total, 1284 patients with CT-confirmed pneumonia associated with COVID-19 were transferred to Tongji Hospital during the study period. Within 72 hours of their admission to hospital, 1159 patients were tested for level of cTnl concentration (Figure 1). Of these patients, $170(14.7 \%)$ patients were positive for an elevated level of
cTnl. In-hospital mortality for these patients was more than 10 -fold higher than in patients without elevated levels of cTnI $(71.2 \%$ [121/170] v. 6.6\% [65/984], $p<0.001$ ) (Figure 2A). Table 1 and Table 2 provide demographic information, clinical symptoms, medical history and treatments as they pertain to the outcome of death in patients with elevated levels of cTnl. Patients who died tended to have more dyspnea $(51.2 \%$ v. $30.6 \%, p=0.02)$, greater need for

A

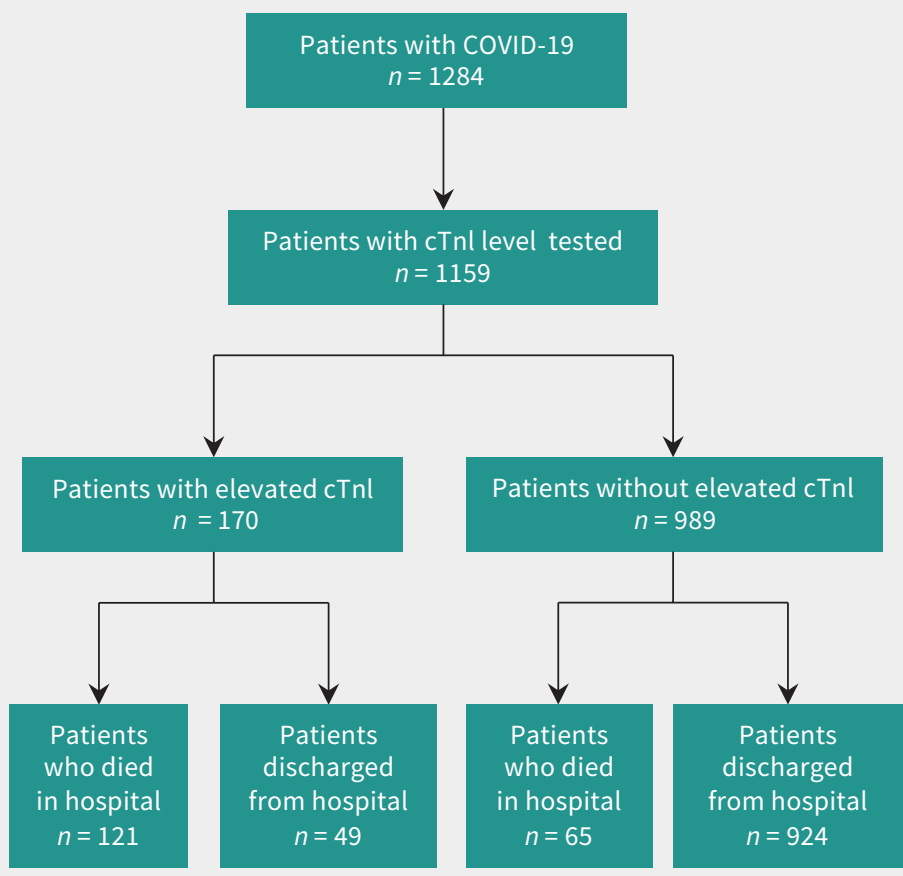

B

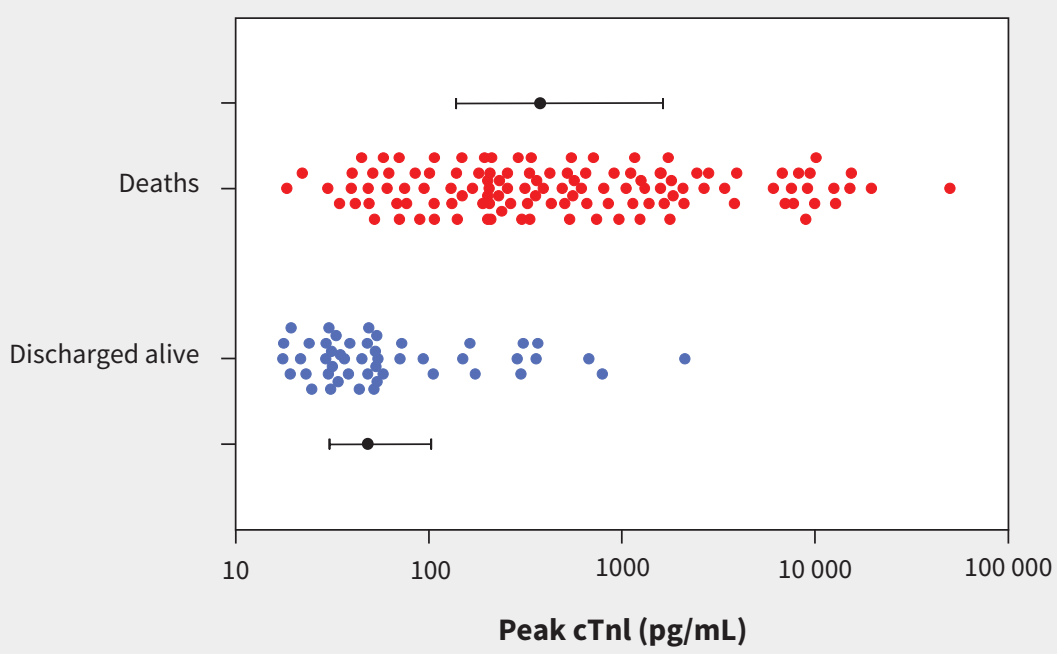

Figure 2: Cardiac injury, death and discharge in coronavirus disease 2019 (COVID-19) patients. (A) Outcomes of death or discharge from hospital of patients based on level of cardiac troponin I (cTnl) at admission. (B) Distribution of cTnl concentrations for the 170 patients who tested positive with outcomes. The median and interquartile range for each outcome group is shown. 
Table 1: Baseline characteristics of patients admitted to hospital with coronavirus disease 2019 who had elevated levels of cardiac troponin I

\begin{tabular}{|c|c|c|c|}
\hline \multirow[b]{2}{*}{ Baseline characteristic } & \multicolumn{2}{|c|}{ No. of patients $(\%)^{\star}$} & \multirow[b]{2}{*}{$\begin{array}{c}p \\
\text { value }\end{array}$} \\
\hline & $\begin{array}{c}\text { Discharged } \\
\text { from hospital } \\
\text { alive } \\
n=49\end{array}$ & $\begin{array}{c}\text { Died in } \\
\text { hospital } \\
n=121\end{array}$ & \\
\hline Age, yr; median $\left(P_{25}, P_{75}\right)$ & $61.5(32,69)$ & $64(24,70)$ & 0.3 \\
\hline Male sex & $16(32.7)$ & $77(63.6)$ & $<0.001$ \\
\hline $\begin{array}{l}\text { Onset of illness, } \dagger \mathrm{d} \text {; } \\
\text { median }\left(P_{25}, P_{75}\right)\end{array}$ & $11(6.5,11.5)$ & $11(7,15)$ & 0.6 \\
\hline \multicolumn{4}{|l|}{ Presenting symptom } \\
\hline Fever & $41(83.7)$ & $101(71.1)$ & 1.0 \\
\hline Cough & $30(61.2)$ & $76(62.8)$ & 0.9 \\
\hline Sputum & $8(16.3)$ & $15(12.4)$ & 0.6 \\
\hline Dyspnea & $15(30.6)$ & $62(51.2)$ & 0.02 \\
\hline Diarrhea & $12(24.5)$ & $25(20.7)$ & 0.7 \\
\hline Chest pain & $3(6.2)$ & $11(9.1)$ & 0.8 \\
\hline \multicolumn{4}{|l|}{ Medical history } \\
\hline Pre-existing CHD & $9(18.4)$ & $21(17.4)$ & 1.0 \\
\hline Hypertension & $30(65.2)$ & $65(53.7)$ & 0.2 \\
\hline Diabetes & $12(24.5)$ & $25(20.7)$ & 0.7 \\
\hline Stroke & $1(2.0)$ & $5(4.1)$ & 0.7 \\
\hline CKD & $4(8.2)$ & $5(4.1)$ & 0.3 \\
\hline Malignant disease & $2(4.1)$ & $9(7.4)$ & 0.5 \\
\hline COPD & $1(2.0)$ & $10(8.3)$ & 0.2 \\
\hline SOFA score on admission $\ddagger$ & $4(2.5,5)$ & $4(3,5.25)$ & 0.3 \\
\hline $\begin{array}{l}\text { APACHE II score on } \\
\text { admission } \ddagger\end{array}$ & $10(7,15)$ & $11.5(9,16)$ & 0.4 \\
\hline
\end{tabular}

Note: $\mathrm{APACHE}$ II = Acute Physiology and Chronic Health Evaluation score, $\mathrm{CHD}=$ coronary heart disease, $\mathrm{CKD}=$ chronic kidney disease, $\mathrm{COPD}=$ chronic obstructive pulmonary disease, COVID-19 = coronavirus disease 2019, $P_{25}=25$ th percentile, $P_{75}=$ 75 th percentile, SOFA = Sequential Organ Failure Assessment score.

*Unless otherwise defined.

tWe defined the onset of illness as the onset of typical symptoms related to COVID-19, such as fever, dry cough and dyspnea.

†The SOFA and APACHE II scores were used to assess organ dysfunction and disease severity. Higher scores indicate a higher rate of organ failure and mortality. We calculated these scores from the data of only 64 patients.

mechanical ventilation (66.9\% v. $4.1 \%, p<0.001)$ and had a higher level of cTnl concentration (393.8 [139.9, 1700] v. 48.5 [30.8, 128.1] $\mathrm{pg} / \mathrm{mL}, p<0.001$ ). Figure $2 \mathrm{~B}$ shows the distribution of $\mathrm{cTn}$ l levels and the outcome of death unadjusted for clinical variables.

Of the 121 patients with COVID-19 and cardiac injury who died, most died of progressive hypoxia due to pneumonia: 56 (46.3\%) with respiratory failure, $23(19.0 \%)$ with septic shock and $6(5.0 \%)$ with acute respiratory distress syndrome. Other causes of death were multiple organ dysfunction syndrome (MODS) in $26(21.5 \%)$, VT/VF in $6(5.0 \%)$ and stroke or intracerebral hemorrhage in $4(3.3 \%)$ patients. After adjusting for age, sex and usage of QT prolongation medication, we found that initial cTnl level (per 10-fold increase, $\mathrm{HR} 1.32,95 \% \mathrm{Cl} 1.06-1.66$ ) and peak CTnI level during illness (per 10-fold increase, HR 1.70, 95\% Cl $1.38-$ 2.10) were associated with poor survival in patients who had cardiac injury at admission to hospital (Table 3). The highest tertile of peak cTnl was associated with greater hazard of mortality ( $\mathrm{HR} 3.78,95 \% \mathrm{Cl} 2.26-6.34)$ than the middle tertile (HR 2.51, $95 \% \mathrm{Cl}$ 1.49-4.22), compared with the lowest tertile. We found that the level of peak cTnl was also an independent predictor of need for invasive ventilatory support (odds ratio $3.02,95 \% \mathrm{Cl}$ 1.92-4.98).

Cardiac rhythm was monitored online in all 170 patients. Initially, arrhythmias were suspected in 60 of these patients, of whom 35 had 12-lead ECG recordings in addition to telemetry monitoring. Of the 60 patients with suspected arrhythmias (based on ECG and telemetry), we changed the diagnosis in 16 to sinus tachycardia episodes with artifact after assessment by cardiac electrophysiologists on our team. Atrial or ventricular arrhythmias were noted in 44 of 170 patients (25.9\%) on 53 occasions (Figure 1). A total of 53 arrhythmia episodes were documented or recorded, because some patients had multiple arrhythmias. Among these 44 patients, 35 had only atrial arrhythmias, 2 had only ventricular arrhythmias and 7 had both atrial and ventricular arrhythmias (Table 4). Ventricular tachycardia/ ventricular fibrillation was fatal in all 6 patients. Of the 25 patients with AT/AF, 4 had a history of atrial fibrillation. Examples of some of these arrhythmias are shown in Figure 3.

On the 35 recorded ECGs, the median QTc interval was prolonged by an average of $45 \mathrm{~ms}$ in those who were on QT-prolonging medications compared with those who were not $(410 \mathrm{~ms}$, quartiles 364, 430 v. $455 \mathrm{~ms}$, quartiles $423,480 \mathrm{~ms}, p=0.01$ ) (Table 5). We found that treatment with QT-prolonging medications was not independently associated with mortality after adjustment for peak cTnl level and other variables (HR 1.04, 95\% Cl 0.69-1.57; Table 2). However, fatal VT/VF occurred in 6 patients, among whom 2 had 12-lead ECGs recorded before death, enabling the QTC interval to be documented. The ECG of 1 of those patients is shown in Appendix 1, Supplementary Figure 1A, available at www.cmaj.ca/lookup/suppl/doi:10.1503/ cmaj.200879/-/DC1), 2 days before death (QTc was 509 ms). This patient was taking arbidol and moxifloxacin, and died of torsade de pointes and VF. The second patient (Appendix 1, Supplementary Figure 1B; QTc was 426 ms, recorded 8 days before death) who died of VF was taking arbidol, moxifloxacin and meropenem.

\section{Interpretation}

In the regional care centre established in Wuhan for patients with severe COVID-19, 14.6\% with CT-proven pneumonia had cardiac injury early during admission to hospital. These patients, when their outcomes were adjudicated after the pandemic, had a mortality rate of $71.2 \%$ compared with those without cardiac injury (6.6\%). The postulate that cardiac injury determines prognosis is further strengthened by the finding that even among those with cardiac injury at admission, the degree of injury during the hospital stay was an independent predictor 
Table 2: Management and course of illness in patients with coronavirus disease 2019 and elevated levels of cardiac troponin I who were discharged from hospital or who died in hospital

\begin{tabular}{|c|c|c|c|}
\hline \multirow[b]{2}{*}{ Characteristic } & \multicolumn{2}{|c|}{ No. of patients $(\%)^{\star}$} & \multirow[b]{2}{*}{$p$ value } \\
\hline & $\begin{array}{c}\text { Discharged from } \\
\text { hospital alive } \\
n=49\end{array}$ & $\begin{array}{l}\text { Died in hospital } \\
\qquad n=121\end{array}$ & \\
\hline Invasive ventilatory support & $2(4.1)$ & $81(66.9)$ & $<0.001$ \\
\hline Peak cTnl, pg/mL; median $\left(P_{25}, P_{75}\right)$ & $48.5(30.8,128.1)$ & $393.8(139.9,1700)$ & $<0.001$ \\
\hline Treatment with antiviral drug & $49(100)$ & $117(96.7)$ & $>1.0$ \\
\hline Arbidol & $30(61.2)$ & $75(67.6)$ & 0.5 \\
\hline Lopinavir & $7(14.3)$ & $9(7.4)$ & 0.2 \\
\hline Oseltamivir & $11(22.5)$ & $12(9.9)$ & 0.05 \\
\hline Ganciclovir & $0(0)$ & $4(3.3)$ & 0.3 \\
\hline Remdesivir & $1(2.0)$ & $0(0)$ & 0.3 \\
\hline Ribavirin & $2(4.1)$ & $28(23.1)$ & 0.003 \\
\hline Treatment with antibiotic & $47(95.9)$ & $116(95.9)$ & $>1.0$ \\
\hline Moxifloxacin/levofloxacin & $36(73.5)$ & $83(68.6)$ & 0.6 \\
\hline Cefuroxime/cefoperazone/cefdinir & $8(16.3)$ & $19(15.7)$ & $>1.0$ \\
\hline Meropenem/imipenem/biapenem & $4(8.2)$ & $33(27.3)$ & 0.03 \\
\hline Azithromycin & $1(2.6)$ & $1(0.8)$ & 0.4 \\
\hline Amoxicillin & $2(4.1)$ & $1(0.8)$ & 0.2 \\
\hline Piperacillin & $0(0)$ & $1(0.8)$ & $>1.0$ \\
\hline Teicoplanin & $0(0)$ & $1(0.8)$ & $>1.0$ \\
\hline Treatment with QT-prolonging medications $†$ & $38(77.6)$ & $89(73.6)$ & 0.7 \\
\hline
\end{tabular}

of death. The mechanism of cardiac injury represented by elevated cTnl levels could be due to viral insult, demand, hypoxia, thrombosis, immune activation, disease severity and subendocardial coronary ischemia. ${ }^{1,7}$ We could not differentiate these mechanisms in our study.

A recent study involving a smaller cohort of patients from another regional COVID-19 care centre in Wuhan, which was published before the pandemic was over, provided findings on the effect of cardiac injury in their patients. ${ }^{1}$ In this study we report on 3 times as many patients and provide detailed characterization of those with cardiac injury. The data set used in the smaller study was from Jan. 20, 2020, to Feb. 10, 2020 , which does not cover the complete course of the virus in Wuhan. When describing the clinical outcomes in patients, Shi and colleagues ${ }^{1}$ listed 319 of 416 patients as remaining in hospital. This constitutes a large number of patients whose clinical outcome was still uncertain, which may explain the lower mortality rate of patients with COVID-19 and cardiac injury included in that study (51.2\%). The mortality rate for our study was $71 \%$, and, in contrast to Shi and colleagues, all patients in our study had either been discharged from hospital or died. In addition, the earlier study did not evaluate arrhythmias. ${ }^{1}$
We have described arrhythmia characteristics in our cohort, including nonfatal arrhythmias such as AF, AT and fPACs, which were the most predominant arrhythmias. Although there was only a small number of documented lethal arrhythmias owing to VT and VF, they are of concern. The common occurrence of cardiac arrhythmias in patients with COVID-19 is strikingly different from what was reported for coronavirus infections due to SARSCoV and Middle East respiratory syndrome (MERS). In a 2003 study involving 196 patients with SARS, a high proportion of whom were critically ill, 8 no substantial cardiac arrhythmias were observed. In another study ${ }^{9}$ involving 121 patients with SARS who were admitted to hospital, cardiac arrhythmias were found in only 1 patient, who had AF. In agreement with our findings, another study involving 138 patients with pneumonia associated with COVID-19 who were admitted to hospital in Wuhan reported cardiac arrhythmias in as many as $16 \%$ of patients and an even higher frequency (44.4\%) among those admitted to ICU, although details were not characterized. ${ }^{4}$

Similar to our findings about VT/VF, a study involving 187 patients in Wuhan reported VT/VF in $5.9 \%$ of patients with SARSCoV-2 infection. ${ }^{2}$

A 2013 study involving patients with MERS with high mortality (up to $60 \%$ ) reported no arrhythmias; ${ }^{10}$ however, a subsequent 


\begin{tabular}{|c|c|c|}
\hline Model & $\begin{array}{l}\text { Unadjusted HR } \\
(95 \% \mathrm{Cl})\end{array}$ & $\begin{array}{c}\text { Adjusted HR } \\
(95 \% \mathrm{CI})\end{array}$ \\
\hline \multicolumn{3}{|l|}{ Cox regression survival analysis } \\
\hline Peak cTnl level (per 10-fold increase) & $1.75(1.43-2.14)$ & $1.70(1.38-2.10)$ \\
\hline \multicolumn{3}{|l|}{ Tertiles of peak cTnl level } \\
\hline Highest tertile (median $1827.1 \mathrm{pg} / \mathrm{mL}$, quartiles 1097.0, 7885.9) & $4.05(2.45-6.70)$ & $3.78(2.26-6.34)$ \\
\hline Middle tertile (median $221.1 \mathrm{pg} / \mathrm{mL}$, quartiles 159.6, 332.5) & $2.53(1.52-4.22)$ & $2.51(1.49-4.22)$ \\
\hline Lowest tertile (median $42.6 \mathrm{pg} / \mathrm{mL}$, quartiles $30.4,54.1$ ) & 1 (Ref.) & 1 (Ref.) \\
\hline Age (per additional year) & NA & $1.01(0.996-1.03)$ \\
\hline Female & NA & $0.74(0.50-1.10)$ \\
\hline Treatment with QT-prolonging medications & NA & $1.04(0.69-1.57)$ \\
\hline \multicolumn{3}{|l|}{ Cox regression survival analysis } \\
\hline Initial cTnl level (per 10-fold increase) & $1.41(1.14-1.76)$ & $1.32(1.06-1.66)$ \\
\hline Age (per additional year) & NA & $1.01(0.996-1.03)$ \\
\hline Female & NA & $0.55(0.37-0.8)$ \\
\hline Treatment with QT-prolonging medications & NA & $1.04(0.69-1.57)$ \\
\hline \multicolumn{3}{|l|}{ Logistic regression model of IMV support } \\
\hline Peak cTnl level (per 10-fold increase) & $2.77(1.82-4.40)$ & $3.02(1.92-4.98)$ \\
\hline Age (per additional year) & NA & $0.99(0.96-1.01)$ \\
\hline Female & NA & $1.17(0.59-2.36)$ \\
\hline Treatment with QT-prolonging medications & NA & $1.10(0.52-2.32)$ \\
\hline
\end{tabular}

Table 4: Arrhythmias in patients with coronavirus disease 2019 and elevated cardiac troponin I

\begin{tabular}{lc} 
Type of arrhythmia & $\begin{array}{c}\text { No. of patients (\%) } \\
\boldsymbol{n}=\mathbf{1 7 0}\end{array}$ \\
Total no. of patients with arrhythmias & $44(25.9)$ \\
With atrial arrhythmias only & $35(20.6)$ \\
AT/AF & $18(10.6)$ \\
fPAC & $16(9.4)$ \\
fPAC and AT/AF & $1(0.6)$ \\
With ventricular arrhythmias only & $2(1.2)$ \\
\hline VT/VF & $1(0.6)$ \\
\hline fPVC and VT/VF & $1(0.6)$ \\
With both atrial and ventricular arrhythmias & $7(4.1)$ \\
\hline AT/AF and VT/VF & $3(1.8)$ \\
\hline AT/AF and fPVC & $3(1.8)$ \\
\hline fPAC and VT/VF & $1(0.6)$ \\
\hline $\begin{array}{l}\text { Note: AT/AF = atrial tachycardia/atrial fibrillation, fPAC = frequent premature atrial } \\
\text { complex, fPVC frequent premature ventricular complex, VT/VF = ventricular } \\
\text { tachycardia/ventricular fibrillation. }\end{array}$
\end{tabular}

smaller study ${ }^{11}$ reported a $15 \%$ incidence of variable tachyarrhythmias and unspecified bradyarrhythmias that were not characterized further, precluding a detailed comparison to COVID-19.

The recent retraction of 2 major COVID-19 studies relating to QT prolongation published in The New England Journal of Medicine ${ }^{12}$ and The Lancet $^{13}$ has added to confusion about the importance of QT-prolonging drugs and mortality in a population of patients with noncardiac injury. Even though a substantial number of the patients included in our study were taking QT-prolonging drugs and their QTc interval was indeed significantly prolonged, we found that this was not independently associated with mortality. The significant QT prolongation in our study, in a population with cardiac injury, in itself is a concern because repolarization heterogeneity and gradients are common and proarrhythmic during cardiac injury. ${ }^{14,15}$ The association of QT-prolonging drugs with mortality should be explored further in larger prospective studies for definitive determination of effect. We suggest that patients with COVID-19 and cardiac injury, especially those starting QT-prolonging drugs, need to be monitored until the outcomes of such studies are known.

\section{Limitations}

We had no access to data on patients without cardiac injury other than mortality. Varying lengths of hospital stay, transfer 
A
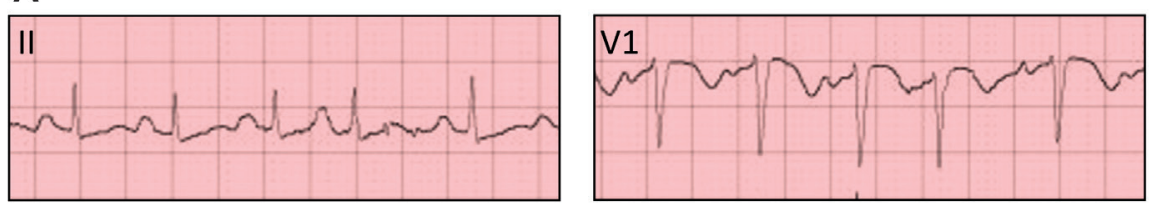

B
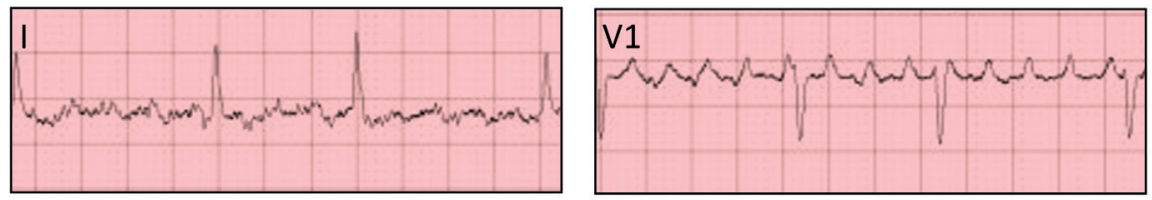

C

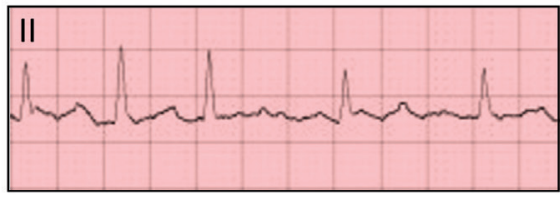

D

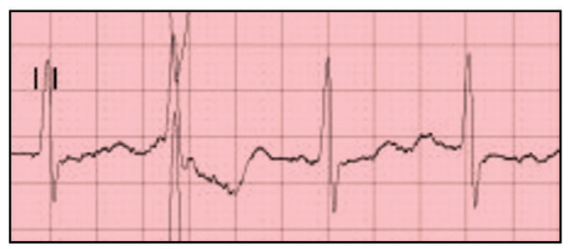

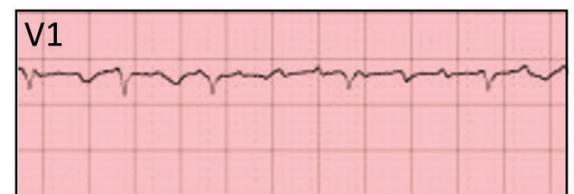

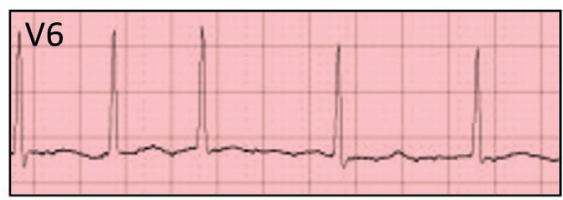

Figure 3: Representative electrocardiograms of arrhythmias in patients with coronavirus disease 2019 (COVID-19) and cardiac injury. (A) Frequent premature atrial complexes. (B) Atrial tachycardia/atrial flutter. (C) Atrial fibrillation. (D) Frequent premature ventricular complexes.

Table 5: Effect of QT-prolonging drugs on electrocardiogram parameters and observed arrhythmias in patients with coronavirus disease 2019 and elevated levels of cardiac troponin I

\begin{tabular}{|c|c|c|c|}
\hline \multirow[b]{2}{*}{ Parameter or type } & \multicolumn{2}{|c|}{ No. of patients (\%) } & \multirow[b]{2}{*}{$\begin{array}{c}p \\
\text { value }\end{array}$} \\
\hline & $\begin{array}{l}\text { Not taking } \\
\text { QT-prolonging } \\
\text { drugs } \\
n=10\end{array}$ & $\begin{array}{l}\text { Taking } \\
\text { QT-prolonging } \\
\text { drugs } \\
n=25\end{array}$ & \\
\hline \multicolumn{4}{|l|}{ Electrocardiogram parameter } \\
\hline PR, ms; median $\left(P_{25}, P_{75}\right)$ & $140(123,176)$ & $142(130,162)$ & 0.9 \\
\hline QRS, ms; median $\left(P_{25}, P_{75}\right)$ & $98(90,107)$ & $142(80,107.5)$ & 0.2 \\
\hline QT, ms; median $\left(P_{25}, P_{75}\right)$ & $340(310,395)$ & $370(305,400)$ & 0.6 \\
\hline QTc, ms; median $\left(P_{25}, P_{75}\right)$ & $410(364.5,429.5)$ & $455(423,479.8)$ & 0.01 \\
\hline $\begin{array}{l}\mathrm{HR} \text {, beats } / \mathrm{min} ; \\
\text { median }\left(P_{25}, P_{75}\right)\end{array}$ & $79(62.5,123.5)$ & $91(75.5,105.5)$ & 0.3 \\
\hline \multicolumn{4}{|l|}{ Type of arrhythmia } \\
\hline fPAC & $3(30.3)$ & $14(56.0)$ & 0.3 \\
\hline $\mathrm{AT} / \mathrm{AF}$ & $3(30.3)$ & $9(36.0)$ & $>0.99$ \\
\hline fPVC & $1(10.0)$ & $3(12.0)$ & 0.6 \\
\hline VT/VF & $0(0.0)$ & $4(16.0)$ & 0.5 \\
\hline \multicolumn{4}{|c|}{ 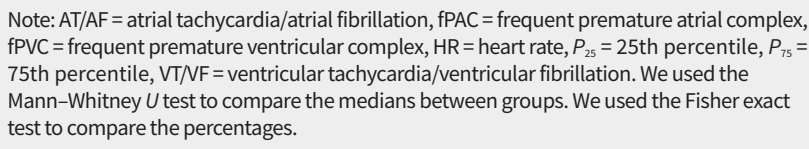 } \\
\hline
\end{tabular}

thresholds, decisions for early versus late intubation and lack of universal arrhythmia assessments affect numerators or denominators and could have affected our estimates. Outcomes at the initial epicentre of the outbreak may not reflect subsequent outcomes; however, a recent report involving 21 patients in Seattle suggests similar serious outcomes for cardiac injury in patients with severe pneumonia associated with COVID-19. ${ }^{16}$

We did not perform routine echocardiographic assessment of left ventricular function in the patients included in our study. Assessment of cardiac injury occurred within the first 72 hours of admission to hospital (which was, on average, $11 \mathrm{~d}$ after disease onset); therefore, we did not evaluate patients with cardiac injury that developed after that period. Our findings apply only to patients who were critically ill and not to those with COVID-19 who were not admitted to hospital.

\section{Conclusion}

We found that cardiac injury assessed early in patients with COVID-19 during admission to a regional hospital was associated with a 10-fold higher probability of death than for those who did not have cardiac injury. We determined that initial cTnl and peak cTnl levels were associated with poor survival, and peak cTnl was a predictor of ventilatory support. Unlike for SARS and MERS, cardiac injury in patients with COVID-19 was associated with arrhythmias. Patients with COVID-19 who start therapy that can prolong repolarization warrant cTnl assessment and monitoring. 


\section{References}

1. Shi S, Qin M, Shen B, et al. Association of cardiac injury with mortality in hospitalized patients with COVID-19 in Wuhan, China. JAMA Cardiol 2020 Mar. 25;e200950 [Epub ahead of print]. doi: 10.1001/jamacardio.2020.0950.

2. Guo T, Fan Y, Chen M, et al. Cardiovascular implications of fatal outcomes of patients with coronavirus disease 2019 (COVID-19). JAMA Cardiol 2020;e201017 [Epub ahead of print]. doi: 10.1001/jamacardio.2020.1017.

3. Zhou F, Yu T, Du R, et al. Clinical course and risk factors for mortality of adult inpatients with COVID-19 in Wuhan, China: a retrospective cohort study. Lancet 2020;395:1054-62.

4. Wang D, Hu B, Hu C, et al. Clinical characteristics of 138 hospitalized patients with 2019 novel coronavirus-infected pneumonia in Wuhan, China. JAMA 2020;323:1061-9.

5. Driggin E, Madhavan MV, Bikdeli B, et al. Cardiovascular considerations for patients, health care workers, and health systems during the coronavirus disease 2019 (COVID-19) pandemic. J Am Coll Cardiol 2020;75:2352-71.

6. Clinical management of severe acute respiratory infection when novel coronavirus (2019-nCoV) infection is suspected: interim guidance, 28 January 2020. Geneva: World Health Organization; 2020. Available: https://apps.who.int/iris/ handle/10665/330893 (accessed 2020 June 12).

7. Chen C, Chen C, Yan JT, et al. Analysis of myocardial injury in patients with COVID-19 and association between concomitant cardiovascular diseases and severity of COVID-19 [article in Chinese]. Zhonghua Xin Xue Guan Bing Za Zhi 2020;48:E008 [Epub ahead of print]. doi: 10.3760/cma.j.cn112148 $-20200225-00123$.
8. Fowler RA, Lapinsky SE, Hallett D, et al.; Toronto SARS Critical Care Group. Critically ill patients with severe acute respiratory syndrome. JAMA 2003;290:367-73.

9. Yu C-M, Wong RS-M, Wu EB, et al. Cardiovascular complications of severe acute respiratory syndrome. Postgrad Med J 2006;82:140-4.

10. Assiri A, McGeer A, Perl TM, et al.; KSA MERS-CoV Investigation Team. Hospital outbreak of Middle East respiratory syndrome coronavirus. N Engl J Med 2013; 369:407-16.

11. Saad M, Omrani AS, Baig K, et al. Clinical aspects and outcomes of 70 patients with Middle East respiratory syndrome coronavirus infection: a single-center experience in Saudi Arabia. Int J Infect Dis 2014;29:301-6.

12. Mehra MR, Desai SS, Kuy S, et al. Retraction: Cardiovascular disease, drug therapy, and mortality in Covid-19. N Eng J Medicine 2020 June 4 [Epub ahead of print]. doi: 10.1056/NEJMoa2007621. 2020.

13. Mehra MR, Desai SS, Ruschitka F, et al. RETRACTED: Hydroxychloroquine or chloroquine with or without a macrolide for treatment of COVID-19: a multinational registry analysis. Lancet 2020 May 22 [Epub ahead of print]. doi: https:// doi.org/10.1016/S0140-6736(20)31180-6.

14. Chauhan VS, Downar E, Nanthakumar K, et al. Increased ventricular repolarization heterogeneity in patients with ventricular arrhythmia vulnerability and cardiomyopathy: a human in vivo study. Am J Physiol Heart Circ Physiol 2006;290:H79-86.

15. Sutton $\mathrm{PM}$, Taggart $\mathrm{P}$, Opthof $\mathrm{T}$, et al. Repolarisation and refractoriness during early ischaemia in humans. Heart 2000;84:365-9.

16. Arentz M, Yim E, Klaff L, et al. Characteristics and outcomes of 21 critically ill patients with COVID-19 in Washington state. JAMA 2020;323:1612-4 [Epub ahead of print]. doi: 10.1001/jama.2020.4326.
Competing interests: Kumaraswamy Nanthakumar is supported by grants from the Canadian Institutes of Health Research, is an Investigator with the Heart and Stroke Foundation and is a consultant for Servier, Biosense Webster, Abbott Laboratories and BlueRock Therapeutics. Stéphane Massé is a consultant for Abbott Laboratories. No other competing interests were declared.

This article has been peer reviewed.

Affiliations: Department of Cardiology (Si, Du, Sun, Liu, Yang), The Third Hospital of Jilin University, Jilin Provincial Molecular Biology Research Center for Precision Medicine of Major Cardiovascular Disease, Changchun, China; Department of Ultrasound (Ni), The Third Hospital of Jilin University, Changchun, China; Institute of Organ Transplantation (Yang), Tongji Hospital, Tongji Medical College, Huazhong University of Science and Technology, Wuhan, China; Intensive Care Unit (Jiang), The Third Hospital of Jilin University, Changchun, China; The Hull Family Laboratory (Massé, J. Nanthakumar, Bhaskaran, K. Nanthakumar), Peter Munk Cardiac Centre, Toronto General Hospital, University of Toronto, Toronto, Ont.; Department of Epidemiology and Biostatistics (Jin), School of Public Health, Jilin University, Changchun, Jilin, China

Contributors: Daoyuan Si and Beibei Du were joint first authors. Daoyuan Si, Beibei Du, Huan Sun, Nan Jiang, Guohui Liu and Kumaraswamy Nanthakumar conceived and designed the study. Bo Yang, Lujia Ni,
Stéphane Massé and Lina Jin extracted the data. Beibei Du, Daoyuan Si, Lina Jin, Jared Nanthakumar and Stéphane Massé analyzed the data and prepared the figures. Beibei Du, Daoyuan Si, Jared Nanthakumar and Kumaraswamy Nanthakumar reviewed the literature and contributed to manuscript drafting. Abhishek Bhaskaran, Jared Nanthakumar and Ping Yang critically reviewed and edited the manuscript. Beibei Du, Daoyuan Si and Kumaraswamy Nanthakumar revised the manuscript for important intellectual content. All of the authors gave final approval of the version to be published and agreed to be accountable for all aspects of the work.

Funding: This work was supported by the grants from Excellent Youth Foundation of Science and Technology of Jilin Province (No. 20180520054JH) and "13th Five-Year" Science Project of Jilin Province Education Department (No. JJKH20190062KJ).

Data sharing: The authors provided the entire database for review for quality and assessment of the veracity of their data to the editor. All reasonable requests for access to the data can be made to the corresponding author, Daoyuan Si (sidaoyuan@jlu.edu.cn).

Accepted: June 9, 2020

Correspondence to: Daoyuan Si, sidaoyuan@jlu.edu.cn; Kumaraswamy Nanthakumar, kumar.nanthakumar@uhn.ca 